\title{
Polyakov Lines in Yang-Mills Matrix Models
}

\author{
Peter Austing \\ Department of Physics, University of Oxford \\ Theoretical Physics, \\ 1 Keble Road, \\ Oxford OX1 3NP, UK \\ E-mail: p.austing@physics.ox.ac.uk \\ Graziano Vernizzi \\ Service de Physique Théorique, CEA/DSM/SPhT Saclay, \\ Unité de recherche associée au CNRS \\ F-91191 Gif-sur-Yvette Cédex, France \\ E-mail: vernizzi@spht.saclay.cea.fr \\ John F. Wheater \\ Department of Physics, University of Oxford \\ Theoretical Physics, \\ 1 Keble Road, \\ Oxford OX1 3NP, UK \\ E-mail: j.wheater@physics.ox.ac.uk
}

\begin{abstract}
We study the Polyakov line in Yang-Mills matrix models, which include the IKKT model of IIB string theory. For the gauge group $S U(2)$ we give the exact formulae in the form of integral representations which are convenient for finding the asymptotic behaviour. For the $S U(N)$ bosonic models we prove upper bounds which decay as a power law at large momentum $p$. We argue that these capture the full asymptotic behaviour. We also indicate how to extend the results to some correlation functions of Polyakov lines.
\end{abstract}

Keywords: Matrix Models, M(atrix) Theories, Nonperturbative Effects. 


\section{Contents}

1. Introduction 1

2. Definition of the models 2

3. Exact calculations for $s u(2)$

4. Upper Bounds on Bosonic Large $p$ Behaviour 8

5. Conclusions

\section{Introduction}

The proposal [1] for a matrix model describing IIB string theory has triggered considerable interest in Yang-Mills matrix models. The models are defined in section 2, and it is the $D=10$ dimensional maximally supersymmetric version which is proposed as the model of string theory. When the gauge group is $S U(2)$ it has been known for some time how to compute the supersymmetric integrals [2-7]. It was believed that the bosonic integrals (in which the fermionic degrees of freedom are omitted) would diverge because of the flat directions in which the matrices all commute, but the authors of [8] computed them analytically for $S U(2)$ and numerically for some other groups, and realised they can converge as long as $D$ is big enough. We established analytically the convergence criteria for the bosonic $S U(N)$ integrals in [9] and for the other gauge groups and supersymmetric integrals in [10], confirming that the partition function does exist in the bosonic case when $D$ is big enough (except for $S U(2)$ and $S U(3), D$ need only be bigger than 2), and that the supersymmetric models exist when $D=4,6$ and 10. For further details, see also [11].

For $S U(N>2)$ it is not known how to compute the integrals exactly, but the authors of [12] used the supersymmetry to deform the partition function into a cohomological theory in which the integrals can be done. Although the relation of this model to the original Yang-Mills model remains unproven, the resulting numbers have been confirmed by very careful numerical calculation for small values of $N[8,13]$, and support a conjecture [14] based on D brane dynamics. The method has also been extended to the other simple groups, and checked numerically for small rank $[15,16]$ and see [17]. Unfortunately, it cannot be extended to observables such as the Polyakov line which break supersymmetry. 
Another important line of research has been into overcoming the considerable difficulties in obtaining numerical results for larger values of $N$ in order to gain understanding of the large $N$ limit. Various simulations of the bosonic $S U(N)$ model have been reported [18-22], and the Polyakov line and Wilson loop have been studied up to $N=768$ in [23]. For the supersymmetric model with $D=4$, the Pfaffian is positive, and these models have been studied up to $N=48[19,24,25]$, and with particular reference to Polyakov lines and Wilson loops [26]. For $D=6$ and $D=10$, the situation is much more difficult since the Pfaffian is complex. Various ways of dealing with this have been tried [27-30]. Meanwhile, supersymmetric random surface, and geometrical approaches have been investigated in [31,32], as have mean field theory and $1 / D$ expansions in [33-38].

The purpose of this paper is to obtain rigorous information about the large $p$ behaviour of the Polyakov line for $S U(N)$ with finite $N$. The scene is set in section 2. In section 3 we derive the exact results for the bosonic and supersymmetric $S U(2)$ models; this is not new but our derivation gives an integral representation which enables the asymptotic behaviour to be calculated very easily. In section 4 we derive upper bounds on the large $p$ behaviour for the Polyakov line in the bosonic theory. These decay polynomially, and as we shall indicate, there is good reason to believe that the upper bounds capture the true behaviour. We also indicate how to extend the results to some correlation functions of Polyakov lines. Section 5 contains a brief discussion.

\section{Definition of the models}

The Yang-Mills matrix integral partition function, which is obtained by dimensionally reducing the Euclidean SSYM action from $D$ down to zero dimensions, is given by

$$
\mathcal{Z}_{D, G}=\int \prod_{\mu=1}^{D} d X_{\mu} \prod_{\alpha=1}^{\mathcal{N}} d \psi_{\alpha} \exp \left(\frac{1}{4} \sum_{\mu, \nu} \operatorname{Tr}\left[X_{\mu}, X_{\nu}\right]^{2}+\frac{1}{2} \operatorname{Tr} \psi_{\alpha}\left[\Gamma_{\alpha \beta}^{\mu} X_{\mu}, \psi_{\beta}\right]\right)
$$

where we adopt the summation convention for repeated indices. The traceless hermitian matrix fields $X_{\mu}$ and $\psi_{\alpha}$ (respectively bosonic and fermionic) are in the Lie algebra $\mathcal{G}$ of the (compact semi-simple) gauge group $G$ and can be written

$$
X_{\mu}=\sum_{a=1}^{g} X_{\mu}^{a} t^{a}, \quad \psi_{\alpha}=\sum_{a=1}^{g} \psi_{\alpha}^{a} t^{a}
$$

where $\left\{t^{a}, a=1, \ldots, g\right\}$ are the generators in the fundamental representation. The $\Gamma_{\alpha \beta}^{\mu}$ are ordinary gamma matrices for $D$ Euclidean dimensions. The model possesses a gauge symmetry

$$
X_{\mu} \rightarrow U^{\dagger} X_{\mu} U, \quad \psi_{\alpha} \rightarrow U^{\dagger} \psi_{\alpha} U, \quad U \in G
$$


and an $S O(D)$ symmetry inherited from the original $D$-dimensional Euclidean symmetry of the SSYM. Although the motivation discussed above leads to a study of the $D=10, S U(N)$ supersymmetric integral, it is useful and illuminating to study several different versions of the model. Firstly by suppressing the fermions we get the bosonic integrals which we will denote by $\mathcal{N}=0$ (ie there are no super-charges) [8]. Secondly the supersymmetric integrals can be written for $D=3,4,6$, and 10, having $\mathcal{N}=2(D-2)$ super-charges. In principle one can integrate out the fermions to obtain

$$
\mathcal{Z}_{D, G}=\int \prod_{\mu=1}^{D} d X_{\mu} \mathcal{P}_{D, G}\left(X_{\mu}\right) \exp \left(-S_{D}(X)\right)
$$

where

$$
S_{D}(X)=-\frac{1}{4} \sum_{\mu, \nu=1}^{D} \operatorname{Tr}\left[X_{\mu}, X_{\nu}\right]^{2}
$$

and the Pfaffian $\mathcal{P}_{D, G}$ is a homogeneous polynomial of degree $\frac{1}{2} \mathcal{N} g$. In this paper we will be concerned with Polyakov line correlation functions of the form

$$
\begin{aligned}
L(P) & =<\operatorname{Tr} \exp \left(i P^{\sigma} X_{\sigma}\right)> \\
& =\int \prod_{\mu=1}^{D} d X_{\mu} \operatorname{Tr} \exp \left(i P^{\sigma} X_{\sigma}\right) \mathcal{P}_{D, G}\left(X_{\mu}\right) \exp \left(\frac{1}{4} \sum_{\mu, \nu} \operatorname{Tr}\left[X_{\mu}, X_{\nu}\right]^{2}\right) .
\end{aligned}
$$

Using the $\mathrm{SO}(\mathrm{D})$ symmetry we can always consider $P^{\sigma}=(p, 0, \ldots)$ and denote the corresponding Polyakov line by $L(p)$.

It is convenient to express the Lie algebra $\mathcal{G}$ using the Cartan-Weyl basis

$$
\left\{H^{i}, E^{\alpha}\right\}
$$

where $i$ runs from 1 to the rank $r$ and $\alpha$ denotes a root. In this basis

$$
\left[H^{i}, H^{j}\right]=0, \quad\left[H^{i}, E^{\alpha}\right]=\alpha^{i} E^{\alpha}
$$

and

$$
\begin{aligned}
{\left[E^{\alpha}, E^{\beta}\right] } & =N_{\alpha \beta} E^{\alpha+\beta} & & \text { if } \alpha+\beta \text { is a root } \\
& =2|\alpha|^{-2} \alpha \cdot H & & \text { if } \alpha=-\beta \\
& =0 & & \text { otherwise. }
\end{aligned}
$$

Here $E^{-\alpha}=\left(E^{\alpha}\right)^{\dagger}$, and the normalisation is chosen such that

$$
\operatorname{Tr} H^{i} H^{j}=\delta^{i j}, \quad \operatorname{Tr} E^{\alpha} E^{\beta}=2|\alpha|^{-2} \delta^{\alpha+\beta}, \quad \operatorname{Tr} H^{i} E^{\alpha}=0 .
$$

We can expand any matrix in the Lie Algebra as

$$
M=\widetilde{M} \cdot H+\sum_{\alpha} \bar{M}^{\alpha} E^{\alpha}
$$


with $\bar{M}^{-\alpha}=\left(\bar{M}^{\alpha}\right)^{*}$ (but for readability's sake we will only use this expansion when necessary). We will denote the set of all roots by $\Delta$, the set of positive roots by $\Delta_{+}$, and the set of simple positive roots by $\Delta_{+}^{*}$; in addition we will denote a simple root by $s$, retaining $\alpha$ for a generic root. The dual weights $\omega_{s}$ satisfy

$$
2 \frac{s \cdot \omega_{s^{\prime}}}{|s|^{2}}=\delta_{s s^{\prime}}
$$

The above definitions (2.7 - 2.12) fix the normalisation of the long root to be $\sqrt{2}$. In the case of $s u(N)$, all roots are of equal magnitude $\sqrt{2}$.

Since the integrand and measure are gauge invariant, we can always make a gauge transformation (2.3) to move $X_{D}$ into the Cartan subalgebra

$$
X_{D}=\lambda_{D} \cdot H
$$

and reduce the integral over $X_{D}$ to an integral over its Cartan modes [15]

$$
\prod_{a=1}^{g} d X_{D}^{a} \rightarrow \Omega_{G}\left(\prod_{i=1}^{l} d \lambda_{D}^{i}\right) \Delta_{G}^{2}\left(\lambda_{D}\right)
$$

where $\Omega_{G}$ is the volume of $G$ and the Weyl measure, given by

$$
\Delta_{G}^{2}\left(\lambda_{D}\right)=\prod_{\alpha \in \Delta_{+}}\left(\lambda_{D} \cdot \alpha\right)^{2},
$$

is the generalisation from $S U(N)$ of the Vandermonde determinant factors.

We will consider mainly the integral (2.6) without fermions so that $\mathcal{N}=0$ and there is no Pfaffian. In the Cartan representation the expression (2.6) takes the form

$$
\begin{aligned}
L(p)= & \int\left(\prod_{i=1}^{r} d \lambda_{D}^{i}\right) \Delta_{G}^{2}\left(\lambda_{D}\right) \prod_{k=1}^{D-1} d X_{k} \operatorname{Tr} \exp \left(i p \lambda_{D} \cdot H\right) \\
& \exp \left(-\sum_{\alpha>0} \frac{\left(\lambda_{D} \cdot \alpha\right)^{2}}{|\alpha|^{2}} \sum_{k=1}^{D-1}\left|\bar{X}_{k}^{\alpha}\right|^{2}+\frac{1}{4} \sum_{j, k=1}^{D-1} \operatorname{Tr}\left[X_{j}, X_{k}\right]^{2}\right)
\end{aligned}
$$

and since the weights in a given representation correspond to the eigenvalues of the Cartan generators, we have

$$
\operatorname{Tr} \exp \left(i p \lambda_{D} \cdot H\right)=\sum_{h} \exp \left(i p \lambda_{D} \cdot h\right),
$$

where $\{h\}$ are the weights. In the case of $s u(N)$, every weight in the fundamental representation is a Weyl transformation of every other weight, so we can use Weyl invariance to write

$$
L(p)=N \int d \lambda_{D} \Delta_{G}^{2}\left(\lambda_{D}\right) d X_{k} \exp \left(i p \lambda_{D} \cdot \omega_{1}-\sum_{\alpha>0} \frac{\left(\lambda_{D} \cdot \alpha\right)^{2}}{|\alpha|^{2}} \sum_{k=1}^{D-1}\left|\bar{X}_{k}^{\alpha}\right|^{2}-S_{D-1}\right)
$$

since $\omega_{1}$ is the first weight in the fundamental representation. 


\section{Exact calculations for $s u(2)$}

In the simplest case of $s u(2)$ it is possible to reduce $L(p)$ to a simple integral representation. This is because there are few enough fields to make good use of the rotation symmetry. ${ }^{1}$ Several authors have used exact calculations of the $s u(2)$ partition function in various contexts [2-7], and integral representations of the eigenvalue density have been given in [40]. In principle one only need take the Fourier transform of the densities given in [40] to obtain the Polyakov line but it is hard to extract the asymptotic behaviour from these representations. The following calculations amount to an alternative representation for the eigenvalue densities from which the asymptotic behaviour of $L(p)$ can be easily determined.

The definitions in section 2 fix the normalisation of the generators, but to avoid any ambiguity in the definition (2.6) we give them explicitly here;

$$
\sigma_{1}=\frac{1}{\sqrt{2}}\left(\begin{array}{ll}
0 & 1 \\
1 & 0
\end{array}\right), \quad \sigma_{2}=\frac{1}{\sqrt{2}}\left(\begin{array}{cc}
0 & -i \\
i & 0
\end{array}\right), \quad \sigma_{3}=\frac{1}{\sqrt{2}}\left(\begin{array}{cc}
1 & 0 \\
0 & -1
\end{array}\right)
$$

so that $H=\sigma_{3}$ and $E^{ \pm 1}=\frac{1}{2}\left(\sigma_{1} \pm i \sigma_{2}\right)$.

\subsection{Bosonic}

There is only one positive root and (2.18) simplifies to

$$
L(p)=2 \int d \lambda_{D} \lambda_{D}^{2} \prod_{k=1}^{D-1} d X_{k} \exp \left(i p \lambda_{D} / \sqrt{2}\right) \exp \left(-\lambda_{D}^{2} \sum_{k=1}^{D-1}\left|\bar{X}_{k}^{1}\right|^{2}-S_{D-1}\right) .
$$

Setting

$$
X_{k}=u_{k} \sigma_{1}+v_{k} \sigma_{2}+\xi_{k} \sigma_{3}, \quad k=1 \ldots d,
$$

where $d=D-1$, we get

$$
L(p)=-2 \frac{d^{2}}{d p^{2}} K(p)
$$

with

$$
K(p)=\int d \lambda e^{i p \lambda} \prod_{k=1}^{D-1} d \xi_{k} d u_{k} d v_{k} \exp \left(-\frac{1}{2} \sum_{i, j=1}^{d} \xi_{i} U_{i j} \xi_{j}-\frac{1}{4} V-\frac{\lambda^{2}}{2} W\right),
$$

where

$$
\begin{aligned}
U_{i j} & =\left(u^{2}+v^{2}\right) \delta_{i j}-\left(u_{i} u_{j}+v_{i} v_{j}\right) \\
V & =u^{2} v^{2}-(u \cdot v)^{2} \\
W & =u^{2}+v^{2} .
\end{aligned}
$$

\footnotetext{
${ }^{1}$ The authors of [39] also managed to extend this to the integrals over the $3 \times 3$ real symmetric matrices.
} 
Integrating out the $d$-dimensional vector $\xi$ gives, up to a constant factor which we will fix later,

$$
K(p)=\int d \lambda d u d v(\operatorname{det} U)^{-\frac{1}{2}} \exp \left(i p \lambda / \sqrt{2}-V / 4-\lambda^{2} W / 2\right)
$$

which, noting that the determinant is $\operatorname{det} U=W^{d-2} V$, we rewrite as

$$
\int d A d B d \lambda \frac{\exp \left(-\lambda^{2} A / 2+i p \lambda / \sqrt{2}-B / 4\right)}{A^{\frac{d-2}{2}} B^{\frac{1}{2}}} J(A, B),
$$

where

$$
\begin{aligned}
J & =\int d u d v \delta(A-W) \delta(B-V) \\
& =\operatorname{const} B^{\frac{d-3}{2}} \theta\left(\frac{A^{2}}{4}-B\right),
\end{aligned}
$$

as one can check by using the rotation invariance. Then integrating out $\lambda$, and scaling $A$ by $p^{2}$, we obtain

$$
K(p)=\frac{(D-2)}{\Gamma\left(\frac{D}{4}-1\right)} p^{4-D} \int_{0}^{\infty} d B B^{\frac{d-4}{2}} e^{-B / 4} \int_{\frac{2 \sqrt{B}}{p^{2}}}^{\infty} d A A^{-\frac{d-1}{2}} e^{-\frac{1}{4 A}},
$$

where we have fixed the constant factor by requiring $L(0)=1$. Differentiating twice with respect to $p$, we obtain terms which vanish like a polynomial from differentiating $p^{4-D}$ and terms which vanish exponentially from differentiating the integral. Then we find

$$
L(p) \sim-2^{D-1} \sqrt{\pi} \frac{\Gamma(D-1)}{\Gamma\left(\frac{D}{4}-1\right)} p^{2-D}
$$

as $p \rightarrow \infty$ for $D>4 .^{2}$

\subsection{Supersymmetric}

For $D=4,6$ and 10, the Pfaffian is given by [13]

$$
\mathcal{P}=\left[\frac{8}{3} \operatorname{Tr}\left[X_{\mu}, X_{\nu}\right]\left[X_{\nu}, X_{\rho}\right]\left[X_{\rho}, X_{\mu}\right]\right]^{\frac{D-2}{2}}=\left[6\left(\lambda^{2} V+\xi_{i} M_{i j} \xi_{j}\right)\right]^{\frac{D-2}{2}},
$$

where

$$
M_{l m}=\delta_{l m} V-2\left(v^{2} u_{l} u_{m}-u \cdot v\left(v_{l} u_{m}+u_{l} v_{m}\right)+u^{2} v_{l} v_{m}\right) .
$$

We note that $M$ and $U$ have the same eigenvectors. Specifically, choosing $d-2$ linearly independent vectors $a$ each perpendicular to $u$ and $v$, we have

$$
\begin{aligned}
U a & =W a, \quad M a=V a \\
U u & =v^{2} u-u \cdot v v, \quad M u=0 \\
U v & =u^{2} v-u \cdot v u, \quad M v=0 .
\end{aligned}
$$

\footnotetext{
${ }^{2}$ For $s u(2)$, the partition function diverges when $D \leq 4$.
} 
The eigenvalues of $U$ on the $u-v$ space are $q_{d-1}$ and $q_{d}$ where $q_{d-1} q_{d}=V$.

Proceeding as in the bosonic case and working in the eigenvector basis of $U$ and $M$ gives

$$
\begin{aligned}
L(p)= & -\frac{d^{2}}{d p^{2}} \int d \lambda e^{i p \lambda / \sqrt{2}} d \xi d u d v\left(\lambda^{2} V+V \sum_{k=1}^{d-2} \xi_{k}^{2}\right)^{\frac{D-2}{2}} \\
& \exp \left(-\frac{1}{2} W \sum_{k=1}^{d-2} \xi_{k}^{2}-\frac{1}{2} q_{d-1} \xi_{d-1}^{2}-\frac{1}{2} q_{d} \xi_{d}^{2}-\frac{1}{4} V-\frac{1}{2} \lambda^{2} W\right) .
\end{aligned}
$$

As before, we can use the rotation symmetry to do the $u$ and $v$ integrals; the Pfaffian terms are pulled down by differentiating with respect to $W$; and then $\xi_{i}$ is integrated out to give

$$
L(p)=\frac{d^{2}}{d p^{2}} \int d \lambda e^{i p \lambda / \sqrt{2}} \int_{0}^{\infty} d A \int_{0}^{A^{2} / 4} d B B^{\frac{D-3}{2}} B^{\frac{D-4}{2}} e^{-B / 4}\left(-\frac{\partial}{\partial A}\right)^{\frac{D-2}{2}} A^{\frac{3-D}{2}} e^{-\lambda^{2} A / 2}
$$

from which the eigenvalue density $\rho(\lambda)$ can be read off. Integrating out $\lambda$ gives

$$
L(p)=-\mathcal{N}_{D} \frac{d^{2}}{d p^{2}} \int_{0}^{\infty} d A A^{2 D-6} e^{-A^{2} / 16}\left(-\frac{\partial}{\partial A}\right)^{\frac{D-4}{2}} A^{\frac{2-D}{2}} e^{-\frac{p^{2}}{4 A}}
$$

where the overall constant factor

$$
\mathcal{N}_{D}=\frac{2^{3(3-D)}(D-3)(D-2) \sqrt{\pi}}{\Gamma\left(\frac{D-3}{2}\right)^{2}}
$$

is fixed by requiring $L(0)=1$. Then, by the saddle point method,

$$
L(p) \sim(-1)^{\frac{D}{2}+1} 2^{3-\frac{5 D}{6}} \sqrt{\frac{\pi}{3}} \mathcal{N}_{D} p^{\frac{4 D-12}{3}} \exp \left(-\frac{3}{16} 2^{\frac{2}{3}} p^{\frac{4}{3}}\right)
$$

as $p \rightarrow \infty$. Note that the asymptotic behaviour is exponential and that the power law terms present in the bosonic case have disappeared. It is tempting to deduce that this is a consequence of the supersymmetry. However it is easy to check that replacing the $\frac{1}{2}(D-2)$ power in the expression for the Pfaffian (3.15) by any positive integer has the same effect of suppressing the power law terms but does not in general come from a supersymmetric theory ${ }^{3}$. The quantity appearing in (3.15) is special in $s u(2)$ because it is proportional to a derivative of the action when expressed in appropriate variables; it is the fact that one of the integrals is then of a total derivative that suppresses the power law.

\footnotetext{
${ }^{3}$ We thank Bergfinnur Durhuus for pointing this out to us.
} 


\section{Upper Bounds on Bosonic Large $p$ Behaviour}

By using the convergence techniques of $[9,10]$ we can obtain an upper bound on the large $p$ behaviour of the Polyakov line for $s u(N)$. We begin by illustrating the method on $s u(2)$ and then on $s u(3)$ before giving the general argument.

\section{$4.1 s u(2)$}

Starting from (3.2) we integrate out $\lambda_{D}$, giving

$$
L(p)=\frac{\sqrt{\pi}}{2} \int \prod_{k=1}^{D-1} d X_{k} Q^{-\frac{3}{2}}\left(2-\frac{p^{2}}{2 Q}\right) \exp \left(-\frac{p^{2}}{8 Q}-S_{D-1}\right),
$$

where

$$
\begin{aligned}
Q & =\sum_{k=1}^{D-1}\left|\bar{X}_{k}^{1}\right|^{2}, \\
S_{D-1} & =-\frac{1}{4} \sum_{j, k=1}^{D-1} \operatorname{Tr}\left[X_{j}, X_{k}\right]^{2},
\end{aligned}
$$

so that a bound on $L(p)$ is given by

$$
|L(p)|<\frac{\sqrt{\pi}}{2} \int \prod_{k=1}^{D-1} d X_{k} Q^{-\frac{3}{2}}\left(2+\frac{p^{2}}{2 Q}\right) \exp \left(-\frac{p^{2}}{8 Q}-S_{D-1}\right) .
$$

In the region of integration which leads to power law behaviour, we must have $Q \rightarrow \infty$ but $S_{D-1}$ bounded, so we will eventually need to examine the flat directions of the $(D-1)$-dimensional action $S_{D-1}$. First though, in any region in which $Q^{-1}>p^{-2+2 \epsilon}$ with $\epsilon$ a small positive parameter, we have exponential behaviour of $L(p)$, so we restrict attention to the region $Q^{-1}<p^{-2+2 \epsilon}$, giving the bound

$$
|L(p)|<\frac{\sqrt{\pi}}{2} \int \prod_{k=1}^{D-1} d X_{k} p^{-3+3 \epsilon}\left(2+\frac{1}{2} p^{2 \epsilon}\right) \exp \left(-\frac{p^{2}}{8 Q}-S_{D-1}\right) .
$$

In order to consider the flat directions, we use the radial variable $R$ defined by

$$
X_{k}=R x_{k}, \quad \sum_{k=1}^{D-1} \operatorname{Tr} x_{k} x_{k}=1
$$

Then certainly $Q<R^{2}$, so we have the bound

$$
|L(p)|<p^{-3+3 \epsilon}\left(2+\frac{1}{2} p^{2 \epsilon}\right) \frac{\sqrt{\pi}}{2} \int \prod_{k=1}^{D-1} d X_{k} \exp \left(-\frac{p^{2}}{8 R^{2}}-R^{4} \hat{S}_{D-1}\right)
$$


where

$$
\hat{S}_{D-1}=-\frac{1}{4} \sum_{j, k=1}^{D-1} \operatorname{Tr}\left[x_{j}, x_{k}\right]^{2} .
$$

To estimate the integral at large $p$ we split the integration domain into two:

$$
\begin{array}{ll}
\mathcal{R}_{1}: & \hat{S}_{D-1} \geq R^{\epsilon-4} \\
\mathcal{R}_{2}: & \hat{S}_{D-1}<R^{\epsilon-4}
\end{array}
$$

where $\epsilon$ is a small positive constant. Since we know that the integrals are convergent the $R$ integral in $\mathcal{R}_{1}$ may be evaluated by the saddlepoint method and we get a contribution to $L(p)$ which decays exponentially at large $p$. On the other hand the contribution from $\mathcal{R}_{2}$ cannot be treated in this way and generates power law behaviour. Bounds on the angular integrals in (4.6) have been computed in [9] and for $s u(2)$, they give

$$
|L(p)|<p^{-3+3 \epsilon}\left(2+\frac{1}{2} p^{2 \epsilon}\right) \frac{\sqrt{\pi}}{2} \int d R \exp \left(-\frac{p^{2}}{8 R^{2}}\right) R^{-D+4+\epsilon^{\prime}},
$$

where $\epsilon^{\prime}$ is another arbitrarily small positive parameter, so that

$$
|L(p)|<\text { const } p^{2-D+\epsilon^{\prime \prime}}
$$

as long as $D \geq 6$.

The difficulty in finding a lower bound is illustrated in this example. In (4.1), we have a positive term and a negative term, and each gives the same upper bound power law behaviour. One can apply the lower bound methods of [10], and find the same power law behaviour as lower bounds for each term (up to arbitrarily small parameters $\epsilon$ again). These terms originate from the Vandermonde factors and, since they each have the same power law behaviour, we cannot rule out cancellation between them.

\section{$4.2 \operatorname{su}(N>2)$}

Completing the square in (2.18) and changing variables from $\lambda$ to $\nu=Q^{\frac{1}{2}} \lambda$ gives,

$$
\begin{aligned}
L(p)= & \int \frac{d \underline{\nu}}{\operatorname{det}(Q)^{\frac{1}{2}}} \prod_{k=1}^{D-1} d X_{k} \prod_{\alpha \in \Delta_{+}}\left(\left(Q^{-\frac{1}{2}} \nu+i p Q^{-1} \omega_{1}\right) \cdot \alpha\right)^{2} \exp \left(-p^{2} \omega_{1} \cdot Q^{-1} \cdot \omega_{1}\right) \\
& \times \exp \left(-\nu \cdot \nu+\sum_{j, k=1}^{D-1} \operatorname{Tr}\left[X_{j}, X_{k}\right]^{2}\right)
\end{aligned}
$$

where

$$
\begin{aligned}
Q_{i j} & =\sum_{\alpha>0} \frac{\alpha^{i} \alpha^{j}}{|\alpha|^{2}} T_{\alpha} \\
T_{\alpha} & =\sum_{k=1}^{d}\left|X_{k}^{\alpha}\right|^{2} .
\end{aligned}
$$


The integrand in (4.11) would be positive definite but for the Weyl determinant factor which we may bound above

$$
\begin{aligned}
\left|\prod_{\alpha \in \Delta_{+}}\left(\left(Q^{-\frac{1}{2}} \nu+i p Q^{-1} \omega_{1}\right) \cdot \alpha\right)^{2}\right| & <\operatorname{const}\left[\sum_{\alpha \in \Delta_{+}}\left\{\left(\alpha^{T} Q^{-\frac{1}{2}} \nu\right)^{2}+p^{2}\left(\alpha^{T} Q^{-1} \omega_{1}\right)^{2}\right\}\right]^{\frac{g-r}{2}} \\
& =\operatorname{const}\left[\nu^{T} Q^{-1} \nu+p^{2}\left(\omega_{1}^{T} Q^{-2} \omega_{1}\right)\right]^{\frac{g-r}{2}}
\end{aligned}
$$

where we have used the relation

$$
\sum_{\alpha \in \Delta_{+}} \alpha \alpha^{T}=\text { const } 1
$$

\subsection{1 $\mathrm{su}(3)$}

Now we will temporarily restrict the algebra to $s u(3)$ and choose the basis $s_{1}=\left(\sqrt{\frac{3}{2}},-\sqrt{\frac{1}{2}}\right), s_{2}=(0, \sqrt{2})$, in which, abreviating $T_{i} \equiv T_{\alpha_{i}}$,

$$
Q^{-1}=\frac{1}{3\left(T_{1} T_{2}+T_{2} T_{3}+T_{3} T_{1}\right)}\left(\begin{array}{cc}
4 T_{1}+T_{2}+T_{3} & -\sqrt{3}\left(T_{2}-T_{3}\right) \\
-\sqrt{3}\left(T_{2}-T_{3}\right) & 3\left(T_{2}+T_{3}\right)
\end{array}\right)
$$

and

$$
\omega_{1}^{T} Q^{-1} \omega_{1}=\frac{2}{9} \frac{4 T_{1}+T_{2}+T_{3}}{\left(T_{1} T_{2}+T_{2} T_{3}+T_{3} T_{1}\right)} .
$$

Since the $T_{a}$ are positive, we can bound any matrix element by

$$
x^{T} Q^{-1} y<\text { const }|x||y| \omega_{1}^{T} Q^{-1} \omega_{1},
$$

which applies in particular to the eigenvalues of $Q^{-1}$. Taking these bounds together with (4.13), we can bound the expression (4.11) for $L(p)$

$$
\begin{aligned}
|L(p)|< & \int d \underline{\nu}\left(\omega_{1}^{T} Q^{-1} \omega_{1}\right)^{r / 2} \prod_{k=1}^{D-1} d X_{k}\left(\omega_{1}^{T} Q^{-1} \omega_{1}\right)^{(g-r) / 2} \sum_{n=0}^{(g-r) / 2} a_{n}(\nu \cdot \nu)^{(g-r) / 2-n} \\
& \left(p^{2} \omega_{1}^{T} Q^{-1} \omega_{1}\right)^{n} \exp \left(-p^{2} \omega_{1}^{T} Q^{-1} \omega_{1}\right) \exp \left(-\nu \cdot \nu+\sum_{j, k=1}^{D-1} \operatorname{Tr}\left[X_{j}, X_{k}\right]^{2}\right),
\end{aligned}
$$

where the $a_{n}$ are the coefficients of the binomial expansion. We can now integrate out $\nu$ giving some new positive coefficients $\tilde{a}_{n}$,

$$
|L(p)|<\int \prod_{k=1}^{D-1} d X_{k} \sum_{n=0}^{(g-r) / 2} \tilde{a}_{n} p^{2 n}\left(\omega_{1}^{T} Q^{-1} \omega_{1}\right)^{n+g / 2} \exp \left(-p^{2} \omega_{1}^{T} Q^{-1} \omega_{1}-S_{D-1}(X)\right) .
$$


We now split the integration region into two parts. In the region in which $\omega_{1}^{T} Q^{-1} \omega_{1} \geq$ $p^{-2+2 \epsilon}$ with $\epsilon$ small but positive, we have exponential behaviour in $p$. We therefore restrict our attention to the region in which

$$
\omega_{1}^{T} Q^{-1} \omega_{1}<p^{-2+2 \epsilon}
$$

Writing $R$ as the radial variable $R^{2}=\widetilde{X}_{\mu}^{i} \widetilde{X}_{\mu}^{i}+\bar{X}_{\mu}^{\alpha} \bar{X}_{\mu}^{-\alpha}$ (with summation over $\mu, i$ and $\alpha$ implied) we have the bound

$$
\omega_{1}^{T} Q^{-1} \omega_{1}>\frac{A}{R^{2}}
$$

where $A$ is a constant, since the $T_{a}$ are positive in (4.16). Inserting (4.20) and (4.21) into $(4.19)$ gives

$$
|L(p)|<\sum_{n=0}^{(g-r) / 2} \tilde{a}_{n} p^{2 n}\left(p^{-2+2 \epsilon}\right)^{n+g / 2} \int \prod_{k=1}^{D-1} d X_{k} \exp \left(-A \frac{p^{2}}{R^{2}}\right) \exp \left(-S_{D-1}(X)\right) .
$$

If $0<R<1$, the behaviour is exponential in $p$. For $R>1$, a bound on the angular part of the integral has been calculated in [9], and we can insert the result directly into (4.22) to obtain

$$
|L(p)|<\sum_{n=0}^{(g-r) / 2} \tilde{a}_{n} p^{-g+\epsilon(2 n+g)} \int_{1}^{\infty} d R \exp \left(-A \frac{p^{2}}{R^{2}}\right) R^{-k_{c}(D-1)-1+\epsilon^{\prime}}
$$

where $\epsilon^{\prime}$ is another arbitrarily small positive number, and

$$
k_{c}(D)=2 r D-D-4 r-\delta_{D, 3} \delta_{r, 2} .
$$

Finally then, we obtain

$$
|L(p)|<\text { const } p^{-k_{c}(D-1)-g+\epsilon^{\prime \prime}}
$$

where $\epsilon^{\prime \prime}$ is a new arbitrarily small but positive number.

These arguments can be extended to some correlation functions of Polyakov lines. Consider

$$
\begin{aligned}
L(p, q)=< & \operatorname{Tr} \exp \left(i p X_{1}\right) \operatorname{Tr} \exp \left(i q X_{1}\right)> \\
=N & \int d \lambda_{D} \Delta_{G}^{2}\left(\lambda_{D}\right) d X_{k} \sum_{h} \exp \left(i p \lambda_{D} \cdot h\right) \sum_{h^{\prime}} \exp \left(i q \lambda_{D} \cdot h^{\prime}\right) \\
& \quad \times \exp \left(-\sum_{\alpha>0} \frac{\left(\lambda_{D} \cdot \alpha\right)^{2}}{|\alpha|^{2}} \sum_{k=1}^{D-1}\left|\bar{X}_{k}^{\alpha}\right|^{2}-S_{D-1}\right) .
\end{aligned}
$$


Clearly the terms with $h=h^{\prime}$ satisfy the same bound as $L(p)$ with $p$ replaced by $p+q$. After completing the square, the cross terms become

$$
\begin{aligned}
L(p)=\int & \frac{d \underline{\nu}}{\operatorname{det}(Q)^{\frac{1}{2}}} \prod_{k=1}^{D-1} d X_{k} \prod_{\alpha \in \Delta_{+}}\left(\left(Q^{-\frac{1}{2}} \nu+i Q^{-1} \sigma\right) \cdot \alpha\right)^{2} \exp \left(-\sigma \cdot Q^{-1} \cdot \sigma\right) \\
& \times \exp \left(-\nu \cdot \nu+\sum_{j, k=1}^{D-1} \operatorname{Tr}\left[X_{j}, X_{k}\right]^{2}\right)
\end{aligned}
$$

where, for example (and specializing to $s u(3)$ ),

$$
\sigma=p \omega_{1}-q \omega_{2}
$$

Introducing an arbitrary constant $0<\beta<1$ we find that

$$
\begin{gathered}
\sigma^{T} Q^{-1} \sigma=\frac{4}{9\left(T_{1} T_{2}+T_{2} T_{3}+T_{3} T_{1}\right)}\left(\beta|\sigma|^{2}\left(T_{1}+T_{2}+T_{3}\right)+T_{1}\left((2 p-q)^{2}-\beta|\sigma|^{2}\right)\right. \\
\left.\quad+T_{3}\left((p-2 q)^{2}-\beta|\sigma|^{2}\right)+T_{2}\left((p+q)^{2}-\beta|\sigma|^{2}\right)\right) .
\end{gathered}
$$

It follows that, in the region

$$
\begin{aligned}
& (2 p-q)^{2}-\beta|\sigma|^{2}=(2 p-q)^{2}-\beta\left(p^{2}+p q+q^{2}\right)>0 \\
& (2 q-p)^{2}-\beta|\sigma|^{2}=(2 q-p)^{2}-\beta\left(p^{2}+p q+q^{2}\right)>0
\end{aligned}
$$

we have

$$
\sigma^{T} Q^{-1} \sigma \geq \beta|\sigma|^{2}\left(\mu_{1}+\mu_{2}\right) \geq \operatorname{const} \frac{\beta|\sigma|^{2}}{R^{2}}
$$

where $\mu_{1,2}$ are the eigenvalues of $Q^{-1}$ so that

$$
\left|x^{T} Q^{-1} y\right| \leq|x||y| \frac{\sigma^{T} Q^{-1} \sigma}{\beta|\sigma|^{2}}
$$

and

$$
|L(p)|<\sum_{n=0}^{(g-r) / 2} \tilde{a}_{n}^{\prime}|\sigma|^{-g}\left(\sigma^{T} Q^{-1} \sigma\right)^{n+g / 2} \int \prod_{k=1}^{D-1} d X_{k} \exp \left(-\sigma^{T} Q^{-1} \sigma\right) \exp \left(-S_{D-1}(X)\right),
$$

where the constants $\tilde{a}_{n}^{\prime}$ depend on $\beta$. Again, unless $\sigma^{T} Q^{-1} \sigma<|\sigma|^{2 \epsilon}$ we get exponential behaviour so proceeding as for $L(p)$ we obtain

$$
L(p, q)<\mathrm{const}|\sigma|^{-k_{c}(D-1)-g+\epsilon^{\prime \prime \prime}} \text {. }
$$

Keeping $\beta$ small but fixed, we see that in the regions not satisfying (4.30) $\sigma$ is almost proportional to a root. Since the roots are weights for the adjoint representation there is screening and it is easy to see that we just get a constant upper bound instead of (4.34). 


\subsection{2 $\operatorname{su}(N>3)$}

We now need to generalise the formula 4.16 for $\omega^{T} Q^{-1} \omega$ to $S U(N)$. First we show that for $Q$ defined as in (4.12),

$$
\operatorname{det} Q=\frac{r+1}{2^{r}} \sum_{\left\{\alpha_{1}, \cdots, \alpha_{r}\right\} \in \mathcal{S}(r)} T_{\alpha_{1}} \cdots T_{\alpha_{r}}
$$

where $\mathcal{S}(n)$ is the set of all n-tuples of positive roots $\alpha$ which are linearly independent. To see this, we use the fact that the determinant is a homogeneous function of the $T_{\alpha}$ of degree $r$. To find the coefficient of $T_{\alpha_{1}} \cdots T_{\alpha_{r}}$ for any choice of the $\left\{\alpha_{1} \cdots \alpha_{r}\right\}$, set $T_{\alpha_{i}}=1, i=1, \cdots, r$ and $T_{\alpha}=0$ otherwise. Then $Q$ becomes

$$
Q_{i j}=\frac{1}{2} \sum_{k=1}^{r} \alpha_{k}^{i} \alpha_{k}^{j}
$$

and

$$
\operatorname{det} Q=2^{-r} \operatorname{det}\left(\alpha_{1}, \cdots, \alpha_{r}\right) \operatorname{det}\left(\alpha_{1}, \cdots, \alpha_{r}\right)^{T} \text {, }
$$

where $\left(\alpha_{1}, \cdots, \alpha_{r}\right)$ is the matrix whose columns are the $\alpha_{i}$. If $\left\{\alpha_{1}, \cdots, \alpha_{r}\right\}$ are linearly dependent then $\operatorname{det} Q=0$. If $\left\{\alpha_{1}, \cdots, \alpha_{r}\right\}$ are linearly independent, then, since for $s u(N)$ any root is a Weyl transformation of any other root and any positive root can be written in terms of simple roots as

$$
\alpha=s_{j}+s_{j+1}+\cdots+s_{k-1}+s_{k}
$$

with $1 \leq j \leq k \leq r$, we can use a series of Weyl transformations and column operations to reduce

$$
\left(\alpha_{1}, \cdots, \alpha_{r}\right) \rightarrow\left( \pm s_{1}, \cdots, \pm s_{r}\right) .
$$

Any minus signs can be dropped since we are only after the square of the determinant. The product of $\left(s_{1}, \cdots, s_{r}\right)$ and its transpose is just the Cartan matrix whose determinant is $r+1$ so

$$
\operatorname{det} Q=\frac{r+1}{2^{r}}
$$

which completes the proof of $(4.35)$.

Now we show that

$$
\omega_{1}^{T} Q^{-1} \omega_{1}=2 \frac{\sum_{s=\left\{\alpha_{1}, \cdots, \alpha_{r-1}\right\} \in \mathcal{S}(r-1)}\left(\frac{n_{s}}{N}\right)^{2} T_{\alpha_{1}} \cdots T_{\alpha_{r-1}}}{\sum_{\left\{\alpha_{1}, \cdots, \alpha_{r}\right\} \in \mathcal{S}(r)} T_{\alpha_{1}} \cdots T_{\alpha_{r}}}
$$

where the $n_{s}$ are non-zero integers. This will allow us to use the same form of bound (4.17) as for $S U(3)$. 
It is helpful to use a basis in which

$$
\omega_{1}=\sqrt{\frac{r}{r+1}}\left(\begin{array}{c}
1 \\
0 \\
\vdots \\
0
\end{array}\right) .
$$

In this basis

$$
\omega_{1}^{T} Q^{-1} \omega_{1}=\frac{r}{r+1} Q^{\perp}{ }_{1,1} / \operatorname{det} Q,
$$

where $Q^{\perp}$ is the matrix of cofactors. Our task then is to compute the cofactor $Q^{\perp}{ }_{1,1}$, and as this is a homogeneous function of the $T_{\alpha}$ of degree $r-1$, we can set $T_{\beta_{(1)}}=\cdots=T_{\beta_{(r-1)}}=1$ and $T_{\alpha}=0$ otherwise, as before. Then

$$
Q^{\perp}{ }_{1,1}=2^{1-r} \operatorname{det} \bar{Q},
$$

where the matrix $\bar{Q}$ is given by

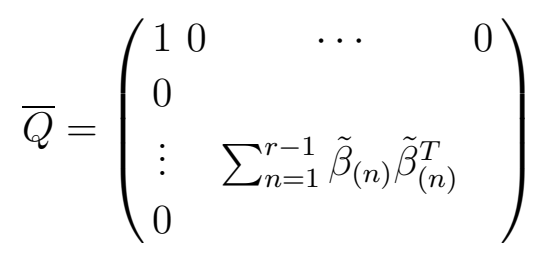

with

$$
\begin{aligned}
& \tilde{\beta}_{(n)}^{1}=0 \\
& \tilde{\beta}_{(n)}^{i}=\beta_{(n)}^{i} \quad i \neq 1 .
\end{aligned}
$$

Proceeding as before we note that

$$
\operatorname{det} \bar{Q}=\operatorname{det}\left(\begin{array}{cccc}
1 & 0 & \cdots & 0 \\
0 & & & \\
\vdots & \tilde{\beta}_{(1)} & \cdots & \tilde{\beta}_{(r-1)} \\
0 & & &
\end{array}\right)^{2}
$$

So, using linear column operations, we can restore the $\tilde{\beta}_{(i)}$ to $\beta_{(i)}$ to get

$$
\operatorname{det}\left(\begin{array}{cccc}
1 & 0 & \cdots & 0 \\
0 & & \\
\vdots & \tilde{\beta}_{(1)} & \cdots & \tilde{\beta}_{(r-1)} \\
0 & &
\end{array}\right)=\sqrt{\frac{r+1}{r}} \operatorname{det}\left(\omega_{1}, \beta_{(1)}, \cdots, \beta_{(r-1)}\right) .
$$

If the $\beta_{(i)}$ are linearly dependent, then $\operatorname{det} \bar{Q}=0$. Using the same procedure as before, we obtain

$$
\operatorname{det}\left(\omega_{1}, \beta_{(1)}, \cdots, \beta_{(r-1)}\right)=\operatorname{det}\left(w \omega_{1}, s_{(1)}, \cdots, s_{(r-1)}\right),
$$


where $w$ is a Weyl transformation, and the $s_{(i)}$ are $r-1$ of the simple roots. We note that this determinant can be zero in some representations of the algebra. For example, in the adjoint representation in which the weights are roots. However, we now show that it is never zero in the fundamental representation. First, since any Weyl transformation is the product of Weyl reflections in roots, we have

$$
w \omega_{1}=\omega_{1}-2 \sum_{i=1}^{r} p_{i} s_{i}
$$

where the $p_{i}$ are integers. In terms of the simple roots, $\omega_{1}$ is given by

$$
\omega_{1}=\frac{1}{r+1}\left[r s_{1}+(r-1) s_{2}+\cdots+s_{r}\right]
$$

so we find

$$
\begin{aligned}
\operatorname{det}\left(w \omega_{1}, s_{(1)}, \cdots, s_{(r-1)}\right) & =\operatorname{det}\left(\frac{q}{r+1} s_{(r)}-2 n s_{(r)}, s_{(1)}, \cdots, s_{(r-1)}\right) \\
& = \pm \frac{n_{s}}{r+1} \operatorname{det}\left(s_{1}, \cdots, s_{r}\right)
\end{aligned}
$$

where $q$ is an integer between 1 and $r, n$ is one of the $p_{i}$, also an integer, and $n_{s}$ is a non-zero integer. Then we have

$$
\operatorname{det} \bar{Q}=\frac{n_{s}^{2}}{r}
$$

and, together with the expression (4.35) for $\operatorname{det} Q$, this completes the result (4.41).

The upper bound for $S U(N)$ now follows in a manner identical to the proof of (4.41). The cofactors of $Q$ take the general form

$$
Q_{i j}^{\perp}=\sum_{s=\left(\alpha_{1} \cdots \alpha_{r-1}\right) \in \mathcal{S}(r-1)} C_{s} T_{\alpha_{1}} \cdots T_{\alpha_{r-1}}
$$

where the $C_{s}$ are positive constants, so that we have again the result (4.17)

$$
x^{T} Q^{-1} y<\text { const }|x||y| \omega^{T} Q^{-1} \omega .
$$

The remainder of the argument follows without modification from the $s u(3)$ case, and we have again

$$
|L(p)|<\operatorname{const}^{-k_{c}(D-1)-g+\epsilon},
$$

where the function $k_{c}$ is given in 4.24, and $\epsilon$ is arbitrarily small.

\section{Conclusions}

For the bosonic $s u(2)$ model the exact calculation shows that asymptotically $L(p) \sim$ $p^{2-D}$ while the upper bound (3.14) demonstrates that this behaviour arises from the flat directions in the action. For larger gauge groups there is unfortunately no exact 
calculation but again the flat directions lead to a power law bound on $L(p)$ at large

$p$ (4.56). In the process of proving the bound we throw away the alternating signs coming from the Vandermonde in (4.11); it is possible that in an exact calculation these would lead to the cancellation of the leading power of $p$. However, as we remarked previously, this does not happen in $s u(2)$ and so there is no reason to suppose that it happens for larger groups. We conclude that $L(p)$ has the asymptotic behaviour

$$
L(p) \sim \operatorname{const}^{-k_{c}(D-1)-g}
$$

at large $p$. Our proof of this bound uses certain properties that are special to the $s u(N)$ Lie algebra but, just as the convergence proofs go through for all gauge groups, we expect that very similar results for $L(p)$ will hold for all the compact Lie groups. This power law property is different from the exponential asymptotic behaviour given by the mean field approximation [34]. The reason is that the mean-field saddle point computation misses the dominant region of integration at large $p$ which is essentially the end-point of the integration domain rather than the saddle point.

Although the asymptotic behaviour of the supersymmetric $s u(2)$ model is qualitatively different, being exponential rather than power law, it is not clear whether this extends to supersymmetric $s u(N)$. In the $s u(2)$ model the power law is suppressed whenever there are fermions in the adjoint representation so this is a feature of $s u(2)$ rather than of supersymmetry. The asymptotic behaviour of the supersymmetric $s u(N)$ models is an open question.

\section{Acknowledgments}

We are grateful to Bergfinnur Durhuus and Thordur Jonsson for useful discussions. PA acknowledges an EPSRC fellowship. GV acknowledges the support of the European network on "Discrete Random Geometries" HPRN-CT-1999-00161 EURO-GRID.

\section{References}

[1] N. Ishibashi, H. Kawai, Y. Kitazawa, and A. Tsuchiya, A Large-N Reduced Model as Superstring, Nucl. Phys. B498 (1997) 467-491, hep-th/9612115.

[2] G. K. Savvidy, Yang-Mills quantum mechanics, Phys. Lett. B159 (1985) 325.

[3] A. V. Smilga, Witten Index Calculation In Supersymmetric Gauge Theory, Nucl. Phys. B266 (1986) 45-57.

[4] A. V. Smilga, Calculation of the Witten Index in extended supersymmetric Yang-Mills theory. (in Russian), Yad. Fiz. 43 (1986) 215-218. 
[5] P. Yi, Witten Index and Threshold Bound States of D-Branes, Nucl. Phys. B505 (1997) 307, hep-th/9704098.

[6] S. Sethi and M. Stern, D-Brane Bound States Redux, Commun. Math. Phys. 194 (1998) 675, hep-th/9705046.

[7] T. Suyama and A. Tsuchiya, Exact results in $N(c)=2$ IIB matrix model, Prog. Theor. Phys. 99 (1998) 321-325, hep-th/9711073.

[8] W. Krauth and M. Staudacher, Finite Yang-Mills Integrals, Phys. Lett. B435 (1998) 350, hep-th/9804199.

[9] P. Austing and J. F. Wheater, The convergence of Yang-Mills integrals, JHEP 02 (2001) 028, hep-th/0101071.

[10] P. Austing and J. F. Wheater, Convergent Yang-Mills matrix theories, JHEP 04 (2001) 019, hep-th/0103159.

[11] P. Austing, Yang-Mills matrix theory, PhD Thesis, hep-th/0108128.

[12] G. Moore, N. Nekrasov, and S. Shatashvili, D-particle bound states and generalized instantons, Commun. Math. Phys. 209 (2000) 77, hep-th/9803265.

[13] W. Krauth, H. Nicolai, and M. Staudacher, Monte Carlo Approach to M-theory, Phys. Lett. B431 (1998) 31-41, hep-th/9803117.

[14] M. B. Green and M. Gutperle, D-instanton partition functions, Phys. Rev. D58 (1998) 046007, hep-th/9804123].

[15] W. Krauth and M. Staudacher, Yang-Mills Integrals for Orthogonal, Symplectic and Exceptional Groups, Nucl. Phys. B584 (2000) 641, hep-th/0004076.

[16] M. Staudacher, Bulk Witten Indices and the Number of Normalizable Ground States in Supersymmetric Quantum Mechanics of Orthogonal, Symplectic and Exceptional Groups, Phys. Lett. B488 (2000) 194, hep-th/0006234.

[17] V. Pestun, $N=4 S Y M$ matrix integrals for almost all simple gauge groups (except $E(7)$ and E(8)), JHEP 09 (2002) 012, [hep-th/0206069].

[18] T. Hotta, J. Nishimura, and A. Tsuchiya, Dynamical aspects of large $N$ reduced models, Nucl. Phys. B545 (1999) 543-575, hep-th/9811220].

[19] J. Ambjorn, K. N. Anagnostopoulos, W. Bietenholz, T. Hotta, and J. Nishimura, Monte Carlo studies of the dimensionally reduced $4 d S U(N)$ super Yang-Mills theory, hep-th/0101084.

[20] S. Horata, H. S. Egawa, and T. Yukawa, Numerical analysis of the double scaling limit in the IIB matrix model, hep-th/0005157. 
[21] N. Kitsunezaki and S. Uehara, Large $N$ structure of IIB matrix model, Prog. Theor. Phys. 105 (2001) 515-524, hep-th/0010038.

[22] N. Kitsunezaki and S. Uehara, Large $N$ behaviors of the IIB matrix model and the regularized Schild models, JHEP 10 (2001) 033, hep-th/0108181.

[23] K. N. Anagnostopoulos, W. Bietenholz, and J. Nishimura, The area law in matrix models for large N QCD strings, Int. J. Mod. Phys. C13 (2002) 555-564, hep-lat/0112035.

[24] J. Ambjorn, K. N. Anagnostopoulos, W. Bietenholz, T. Hotta, and J. Nishimura, Large $N$ dynamics of dimensionally reduced $4 d S U(N)$ super Yang-Mills theory, JHEP 07 (2000) 013, hep-th/0003208].

[25] Z. Burda, B. Petersson, and J. Tabaczek, Geometry of reduced supersymmetric $4 d$ Yang-Mills integrals, hep-lat/0012001.

[26] J. Ambjorn, K. N. Anagnostopoulos, W. Bietenholz, F. Hofheinz, and J. Nishimura, On the spontaneous breakdown of Lorentz symmetry in matrix models of superstrings, Phys. Rev. D65 (2002) 086001, hep-th/0104260.

[27] J. Ambjorn, K. N. Anagnostopoulos, W. Bietenholz, T. Hotta, and J. Nishimura, Monte Carlo studies of the IIB matrix model at large N, JHEP 07 (2000) 011, hep-th/0005147.

[28] J. Nishimura and G. Vernizzi, Brane world generated dynamically from string type IIB matrices, Phys. Rev. Lett. 85 (2000) 4664-4667, hep-th/0007022.

[29] J. Nishimura and G. Vernizzi, Spontaneous breakdown of Lorentz invariance in IIB matrix model, JHEP 04 (2000) 015, hep-th/0003223].

[30] G. Vernizzi and J. F. Wheater, Rotational symmetry breaking in multi-matrix models, Phys. Rev. D66 (2002) 085024, hep-th/0206226.

[31] P. Bialas, Z. Burda, B. Petersson, and J. Tabaczek, Large N limit of the IKKT matrix model, Nucl. Phys. B592 (2001) 391-407, hep-lat/0007013.

[32] Z. Burda, J. Erdmann, B. Petersson, and M. Wattenberg, Exotic trees, Phys. Rev. E67 (2003) 026105, cond-mat/0207459.

[33] H. Aoki, S. Iso, H. Kawai, Y. Kitazawa, and T. Tada, Space-time structures from IIB matrix model, Prog. Theor. Phys. 99 (1998) 713-746, hep-th/9802085.

[34] S. Oda and F. Sugino, Gaussian and mean field approximations for reduced Yang-Mills integrals, JHEP 03 (2001) 026, hep-th/0011175.

[35] F. Sugino, Gaussian and mean field approximations for reduced 4 d supersymmetric Yang-Mills integral, hep-th/0105284. 
[36] J. Nishimura and F. Sugino, Dynamical generation of four-dimensional space-time in the IIB matrix model, JHEP 05 (2002) 001, hep-th/0111102.

[37] H. Kawai, S. Kawamoto, T. Kuroki, T. Matsuo, and S. Shinohara, Mean field approximation of IIB matrix model and emergence of four dimensional space-time, Nucl. Phys. B647 (2002) 153-189, hep-th/0204240.

[38] H. Kawai, S. Kawamoto, T. Kuroki, and S. Shinohara, Improved perturbation theory and four-dimensional space-time in IIB matrix model, Prog. Theor. Phys. 109 (2003) 115-132, hep-th/0211272.

[39] G. M. Cicuta, L. Molinari, and G. Vernizzi, Yang-Mills integrals, J. Phys. A35 (2002) L51-L59, hep-th/0109160.

[40] W. Krauth, J. Plefka, and M. Staudacher, Yang-Mills Integrals, Class. Quant. Grav. 17 (2000) 1171, hep-th/9911170].

[41] W. Krauth and M. Staudacher, Eigenvalue distributions in Yang-Mills integrals, Phys. Lett. B453 (1999) 253-257, hep-th/9902113]. 